\title{
Die Ökonomisierung der Medizin
}

\section{Liebe Kolleginnen und Kollegen, liebe Mitstreiterinnen und Mitstreiter im Gesundheitsmarkt}

In Anlehnung an den Spruch «Ohne Geld keine Musik» könnte man sagen «Ohne Geld keine Medizin». In den meisten Gesellschaften erhalten Medizinerinnen und Mediziner Geld für ihre erbrachten Leistungen, in einigen Regionen der Welt erhielten Mediziner eine Belohnung, wenn sie die Menschen gesund hielten.

Dass Geld und Medizin etwas miteinander zu tun haben, ist nichts Neues. Fast niemand stellt in Abrede, dass die Medizin mit der Ökonomie, der Verteilung knapper Güter, untrennbar verknüpft ist. Gibt die Gesellschaft mehr für die Medizin aus, kann sie, wenn sie haushälterisch mit Geld umgeht, weniger für Bildung, den Verkehr oder die Landesverteidigung ausgeben.

Neu ist, zumindest in unserer Wahrnehmung, dass immer öfter von der Ökonomisierung der Medizin gesprochen wird, wobei nicht immer klar wird, was mit diesem Begriff genau gemeint ist. Oft ist er aber negativ oder kritisch konnotiert im Sinne von «einige Medizinerinnen und Mediziner verdienen sich eine goldene Nase». Gut bezahlt werden nur technische Leistungen, das Gespräch mit Patientinnen und Patienten ist wirtschaftlich nicht rentabel. Unnötige Untersuchungen oder Operationen werden durchgeführt, um das eigene Einkommen oder die Einnahmen des Krankenhauses aufzubessern. Wer möglichst viele Patientinnen und Patienten operiert, erhält einen «Bonus» für die vielen, teilweise auch unnötigen Eingriffe, und zuweisende Ärztinnen und Ärzte erhalten sogenannte Kick-back-Zahlungen. Das sind Aussagen, die im Zusammenhang von Medizin und Ökonomisierung immer wieder zu hören sind. Wahrscheinlich wäre der Begriff «Kommerzialisierung» - ein Vorgang, bei dem aus Dingen wie der Gesundheit und Krankheit mehr und mehr ein Geschäft gemacht wird - treffender als der der Ökonomisierung.

Wir haben verschiedene Personen gebeten - Medizinerinnen und Mediziner, Standespolitikerinnen und -politiker, Ökonomen, Patientinnen und Patienten, Schriftstellerinnen und Schriftsteller - ihre Gedanken zum Thema Ökonomisierung in der Medizin in Worte zu fassen.

Wie erwartet, sind die Meinungen dazu nicht einheitlich. Sie können sich nun selbst eine Meinung bilden. Wir wünschen eine informative Lektüre und ermutigen Sie, Kommentare zu verfassen - entweder als E-mail oder auf der nachfolgenden «leeren» Seite, die Sie dann fotografieren/scannen und per Mail an die Redaktion schicken (redaktion.praxis@hogrefe.ch)-die Kommentare werden in einer der nächsten Ausgaben oder auf der Website der «Praxis» veröffentlicht.

PD Dr. med. Markus Schneemann

Chefarzt Innere Medizin

Spitäler Schaffhausen

Markus.Schneemann@spitaeler-sh.ch

Prof. em. Dr. med. Johann Steurer

Universitätsspital Zürich

johann.steurer@usz.ch

Prof. Dr. med. Paolo M. Suter

Leitender Arzt an der Klinik und Poliklinik für Innere Medizin

Universitätsspital Zürich

paolo.suter@usz.ch 